\title{
Selection of singular solutions in non-local transport equations
}

\author{
J. Eggers ${ }^{1}$, M. A. Fontelos ${ }^{2}$
}

Abstract. We consider two different non-local, and non-linear transport equations, both of which form singularities in finite time, starting from smooth initial conditions. The first,

$$
\theta_{t}=D^{(\gamma)}(\theta) \theta_{x}
$$

is a non-local version of the inviscid Burgers' equation, which is hyperbolic and forms a shock in finite time; $D^{(\gamma)}$ denotes the fractional derivative, which for $\gamma=0$ is the Hilbert transform: $D^{(0)}(\theta)=H(\theta)$. We show that singular solutions of the non-local equation for $\gamma<1$ connect to the hierarchy of shock solutions of Burgers' equation, which are obtained for $\gamma=1$. The second equation,

$$
\theta_{t}-\delta(\theta H(\theta))_{x}-(1-\delta) H(\theta) \theta_{x}=0
$$

is a simplified version of a class of ill-posed problems arising in the theory of vortex sheets and water waves, which are known to exhibit a weak curvature singularity in finite time, known as "Moore's singularity". The linearized form of (2) allows for a continuous family of curvature singularities, with the scaling exponent $\alpha$ as a parameter, each of which is identical to those arising in Moore's singularity. By considering the stability of each singularity, we are able to determine which exponent is selected, and show that its value depends on the parameter $\delta$. 
School of Mathematics, University of Bristol, University Walk, Bristol BS8 1TW, United Kingdom ${ }^{1}$

Instituto de Ciencias Matemáticas, (ICMAT, CSIC-UAM-UCM-UC3M), C/ Serrano 123, 28006 Madrid, Spain²

\section{Introduction}

The formation of singularities in non-linear PDE's is characterized by self-similar solutions $[16,17]$, such that as the singularity is approached, the size of the dependent and independent variables are power laws in the time distance $t^{\prime}=t_{0}-t$ to the singularity. In the present paper, we look for similarity solutions to (1),(2) of the form

$$
\theta=\theta_{0}+t^{\prime \alpha} \Theta(\xi), \quad \xi=x^{\prime} / t^{\prime \beta},
$$

where $\alpha$ and $\beta$ are as-yet undetermined scaling exponents, $x^{\prime}=x-x_{0}$ (with $x_{0}$ the point where singularity forms), and $\theta_{0}$ is a constant. In many cases, the values of the scaling exponents $\alpha, \beta$ is determined from a balance of different terms in the equation [17]. However, in the case of (1) and (2), to leading order the balance which determines (3) consists of two terms only. As a result, while $\beta$ can be computed (as a function of $\alpha$ ), the other exponent $\alpha$ remains a free parameter. This particular class of problems, in which the structure of the equation itself does not specify the exponent, is known as self-similarity of the second kind $[4,3]$. Rather, an additional condition on the regularity of the solution leads to selection of a particular scaling exponent.

Many fluid mechanics problems of long standing lead to equations non-local in character [27], and numerous non-local model equations have been put forward to study such problems $[10,11,8,12,21,22]$. While self-similarity of the second kind has been explored in many examples in the case of local PDE's, analytical insight into the mechanism of exponent selection has been scarce for non-local problems. The perhaps most well-known example of such a selection problem is Moore's singularity of vortex sheets $[29,2,15,30]$. There is strong numerical evidence that the curvature of a vortex sheet diverges like $t^{\prime-1 / 2}[29,28,23]$, yet this observation has never been explained without making ad-hoc assumptions, based in particular on the analytic structure of solutions in the complex plane. Weakly nonlinear systems deduced from a Hamiltonian formulation of vortex sheets (and, in general, the evolution of interfaces between potential flows with different densities) have been studied by means of analytic continuation techniques in $[24,25]$ and result in the development of Moore-type singularities.

In the present paper we consider two different model equations, to illustrate the existence of two different mechanisms of selection. The first equation is the fractional transport equation (1), which interpolates continuously between two cases studied previously. The fractional derivative $D^{(\gamma)}$ is defined as the non-local operator

$$
D^{(\gamma)}(f)(x)=P_{\gamma} \int_{-\infty}^{\infty} \frac{f(y) \operatorname{sign}(x-y)}{|x-y|^{1+\gamma}} d y
$$


where $P_{\gamma}=-1 /\left(2 \sin \frac{\gamma \pi}{2} \Gamma(-\gamma)\right)$ and the integral is understood in the principal value sense. This definition guarantees that the Fourier transform satisfies

$$
\mathcal{F}\left(D^{(\gamma)}(f)\right)(q)=-i \operatorname{sign}(q)|q|^{\gamma} \mathcal{F}(f)(q),
$$

so that

$$
D^{(0)}(f)(x)=H(f)(x)=\frac{1}{\pi} \int_{-\infty}^{\infty} \frac{f(y)}{x-y} d y
$$

is the Hilbert transform and $D^{(1)}(f)=-f_{x}$ is (minus) the ordinary derivative.

This means that for $\gamma=1,(1)$ is $\theta_{t}+\theta_{x}^{2}=0$. Taking the first derivative and putting $u=\theta_{x}$, this is the kinematic wave equation

$$
u_{t}+2 u u_{x}=0,
$$

which generically leads to shock solutions [26, 17]. The self-similar properties of these shock solutions have been studied in detail in [31, 16], and have recently been extended to higher dimensions [18]. On the other hand, taking the limit $\gamma \rightarrow 0,(6)$ is connected to the non-local equation

$$
\theta_{t}=H(\theta) \theta_{x},
$$

which has been studied previously in [12]. Below we will show that exponent selection in (7) can be understood by a regularity condition, which is an extension of the corresponding condition for (6).

While (1) is hyperbolic in character, its singularity resulting from the crossing of characteristics, we will see that (2) is elliptic, and the formation of its singularity is related closely to its ill-posed character. In [12], (2) has been studied both theoretically and by full numerical simulation. In particular, it was shown that $\alpha=1 / 2$ in the case $\delta=1$, which leads to a solution identical to that for Moore's singularity, which is observed to exhibit a $t^{-1 / 2}$ curvature singularity. However, there is strong numerical evidence that for $0<\delta<1, \alpha$ is a smooth function of $\delta$, and falls far below the "generic" value of $\alpha=1 / 2$ observed for vortex sheets [29, 23]. Therefore, a new mechanism for the selection of $\alpha$ needs to be found. In this paper we will provide an analytical method to compute $\alpha$ as a function of $\delta$, and confirm the result by comparison with numerical simulations.

For $\delta=0$, (2) becomes identical to (1) with $\gamma=0$, and thus to (7). However, this represents a singular limit [12]; in particular, for $\delta=0$ the value of the maximum of the profile remains at the same value $\theta_{0}$, while for $\gamma>0$ it is the sum of $\theta_{0}$ plus a nontrivial profile and hence becomes time dependent. Indeed, we will show below that (1) has the scaling exponent $\alpha \approx 1.181 \ldots$ for $\gamma=0$, while in the limit $\delta \rightarrow 0,(2)$ has the scaling exponent $\alpha=0.1767 \ldots$

In the following section, we first consider the Burgers-type equation (1), and in the next, the Moore-type equation (2). We close with a discussion of our results and of future perspectives. 
Selection in non-local equations

\section{Burgers-type equation}

\subsection{Preliminaries}

We will be looking for symmetric solutions of (1) for which $\theta$ is even. Then the fractional derivative becomes

$$
D^{(\gamma)}(f)(x)=-\frac{1}{2 \sin \frac{\gamma \pi}{2} \Gamma(-\gamma)} \int_{0}^{\infty} f(y)\left(\frac{\operatorname{sign}(x-y)}{|x-y|^{1+\gamma}}+\frac{1}{|x+y|^{1+\gamma}}\right) d y,
$$

so that for large $y$ the kernel behaves like

$$
\frac{\operatorname{sign}(x-y)}{|x-y|^{1+\gamma}}+\frac{1}{|x+y|^{1+\gamma}} \approx \frac{2(1+\gamma) x}{y^{2+\gamma}} .
$$

This means the integral converges if $f(y)$ grows more slowly than $y^{1+\gamma}$. For the case $\gamma=0$ and symmetric $f,(8)$ becomes

$$
D^{(0)}(f)(x) \equiv H(f)(x)=\frac{2 x}{\pi} \int_{0}^{\infty} \frac{f(y)}{x^{2}-y^{2}} d y,
$$

which converges if $f$ grows more slowly than linear.

For increasing $\gamma$, the integrand (4) becomes more and more singular. It is therefore advantageous to integrate by parts to obtain

$$
\begin{aligned}
& D^{(\gamma)}(f)(x)=\frac{P_{\gamma}}{\gamma} \int_{0}^{\infty} f^{\prime}(y)\left[(x+y)^{-\gamma}-|x-y|^{-\gamma}\right] d y= \\
& -\frac{P_{\gamma}}{\gamma(1-\gamma)} \int_{0}^{\infty} f^{\prime \prime}(y)\left[\operatorname{sign}(x-y)|x-y|^{1-\gamma}+(x+y)^{1-\gamma}\right] d y .
\end{aligned}
$$

In the limit $\gamma=1,-P_{\gamma} /(\gamma(1-\gamma))=-1 / 2$, and the kernel in (10) vanishes for $y>x$, and so

$$
D^{(1)}(f)(x)=-\frac{1}{2} \int_{0}^{x} 2 f^{\prime \prime}(y)=-f^{\prime}(x)
$$

as expected. In the case $\gamma=0$ one can either take the limit in (10) or work directly from (9) to obtain

$$
D^{(0)}(f)(x)=\frac{1}{\pi} \int_{0}^{\infty} f^{\prime \prime}(y)[(x+y) \ln (x+y)+(x-y) \ln |x-y|] d y .
$$

\subsection{Similarity equation}

The singularity develops at a local maximum, whose value $\theta_{0}$, set by the initial condition, remains constant. Inserting (3) into (1), a balance is achieved for $\beta=(1+\alpha) /(1+\gamma)$, and the similarity equation becomes

$$
\alpha \Theta-\frac{(1+\alpha) \xi}{1+\gamma} \Theta_{\xi}=-D^{(\gamma)}(\Theta) \Theta_{\xi}
$$

We solve (12) for a symmetric profile on the interval $\xi \in[0, \infty[$. The singular solution (3) has to agree with a time-independent outer solution, which leads to the matching 
condition [17] $\theta \propto \xi^{\frac{\alpha(1+\gamma)}{1+\alpha}}$ on the behaviour of $\theta$ in the limit of large $\xi$. In fact, observing that (12) is invariant under the transformation

$$
\theta(\xi)=a \widetilde{\theta}\left(\frac{\xi}{a^{1+\gamma}}\right),
$$

we can always rescale to adjust the prefactor such that the asymptotic behaviour for large arguments is

$$
\theta(\xi)=-\xi^{\frac{\alpha(1+\gamma)}{1+\alpha}} \equiv-\xi^{\nu}, \quad \xi \rightarrow \infty .
$$

With this normalisation, the solution to (12) is unique. In order to provide boundary conditions for the numerical solution of (12) it is useful to calculate the next order in the expansion for large arguments:

$$
\theta(\xi)=-\xi^{\nu}-\nu B(\gamma, \nu) \xi^{2 \nu-\gamma-1}+\ldots, \quad \xi \rightarrow \infty
$$

where $B(\gamma, \nu)$ is given in Appendix A.

\section{3. $\gamma=1$}

In the case $\gamma=1$, for which (1) becomes the kinematic wave equation,(12) can be solved exactly [19]; the similarity equation is

$$
-\alpha \Theta+\frac{(1+\alpha) \xi}{2} \Theta_{\xi}+\Theta_{\xi}^{2}=0 .
$$

Differentiating with respect to $\xi$ and putting $U=\Theta_{\xi}$ this becomes

$$
\frac{1-\alpha}{2} U+\frac{(1+\alpha) \xi}{2} U_{\xi}+2 U U_{\xi}=0
$$

which has solution

$$
\xi=-2 U-C U^{\frac{\alpha+1}{\alpha-1}},
$$

with an arbitrary constant $C$. For the solution to be regular and one-to-one, we need $(\alpha+1) /(\alpha-1)=2 i+3$, where $i$ is a non-negative integer, and so the exponents become

$$
\alpha=\frac{i+2}{i+1}, \quad \beta=\frac{2 i+3}{2 i+2} .
$$

The generic (stable) case is $i=0$ and $\alpha=2, \beta=3 / 2[16,17]$. For $i=1, \ldots$, higher order, unstable solutions are generated, for example $\alpha=3 / 2$ for $i=1$.

To obtain $\Theta(\xi)$, we observe that $\Theta_{\xi}=\Theta_{U} U_{\xi}=\Theta_{U}\left(\xi_{U}\right)^{-1}$, so that

$$
U \xi_{U}=\Theta_{U}=-2 U-(2 i+3) C U^{2 i+3} \text {. }
$$

Integrating, we have

$$
\xi=-2 U-C U^{2 i+3}, \quad \Theta=-U^{2}-\frac{(2 i+3) C U^{2 i+4}}{2 i+4},
$$

which corresponds to what was found in [19]. The normalisation $\Theta \approx-\xi^{\frac{2 i+4}{2 i+3}}$ for large $\xi$ corresponds to $C=\left(\frac{2 i+3}{2 i+4}\right)^{2 i+3}$. 


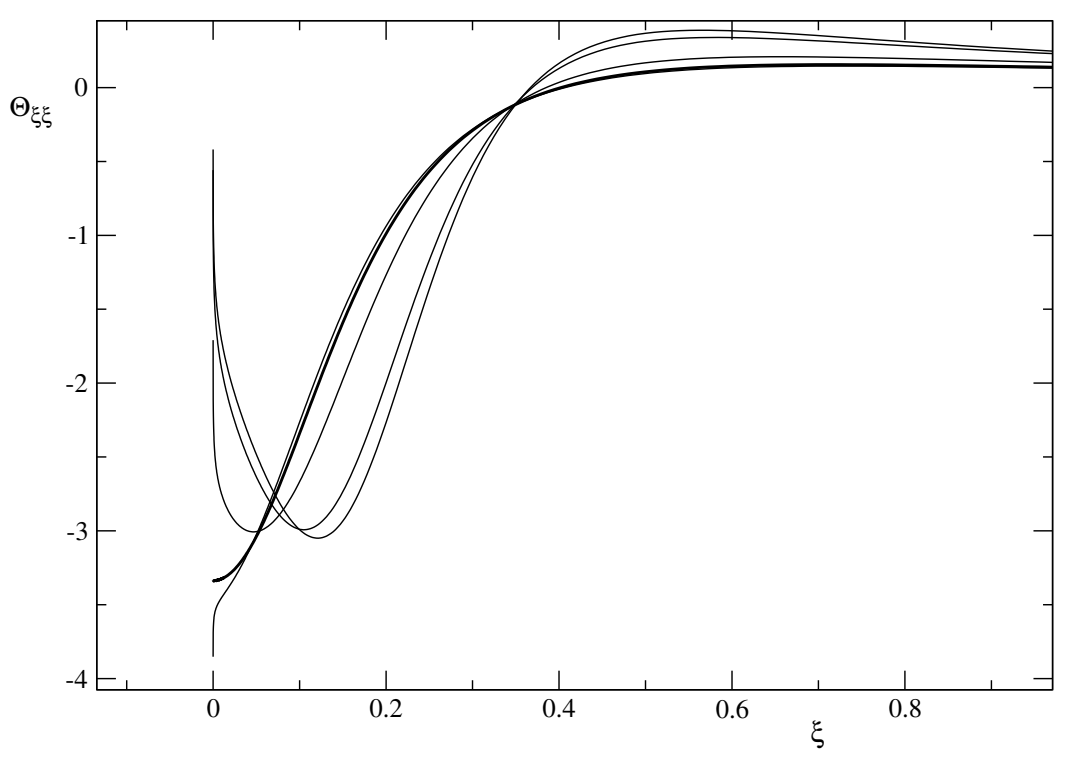

Figure 1. The second derivative $\Theta_{\xi \xi}$ for various values of $\alpha$. The critical value $\alpha=1.177$, for which the profile is smooth at the origin, corresponds to the heavy line.

\subsection{Exponent selection}

To solve (12) for general $\gamma$, we use Newton's method, discretizing the integral representation (4). For values of $\gamma$ close to one, we choose the second form of (10), such that the integrand is much less singular. Trying to solve (12) using Newton's method, the second derivative is very noisy. Therefore, we take the derivative of (12) to solve

$$
r(\xi)=\frac{\alpha \gamma-1}{1+\gamma} \Theta_{\xi}-\frac{1+\alpha}{1+\gamma} \xi \Theta_{\xi \xi}+\left(D^{(\gamma)}(\Theta) \Theta_{\xi}\right)_{\xi}=0 .
$$

Now the profile is smooth for $\xi>0$, but has a singularity at the origin of the form $\Theta=a \xi^{\beta}$. This can be understood noting that to leading order near the origin,

$$
D^{(\gamma)}(\Theta)=-2 P_{\gamma}(1+\gamma) \int_{0}^{\infty} \frac{\Theta(y)}{y^{2+\gamma}} x
$$

Balancing the leading-order terms in (12) we find that

$$
\alpha-\beta \frac{1+\alpha}{1+\gamma}=2 \beta(1+\gamma) P_{\gamma} \int_{0}^{\infty} \frac{\Theta(y)}{y^{2+\gamma}} d y
$$

In other words, for the solution to be regular (i.e. $\beta=2$ ), the constraint

$$
\int_{0}^{\infty} \frac{\Theta(y)}{y^{2+\gamma}} d y=-\frac{2+\alpha(1-\gamma)}{4(1+\gamma)^{2} P_{\gamma}}=\frac{2(2+\alpha(1-\gamma)) \sin \frac{\gamma \pi}{2} \Gamma(-\gamma)}{4(1+\gamma)^{2}}
$$




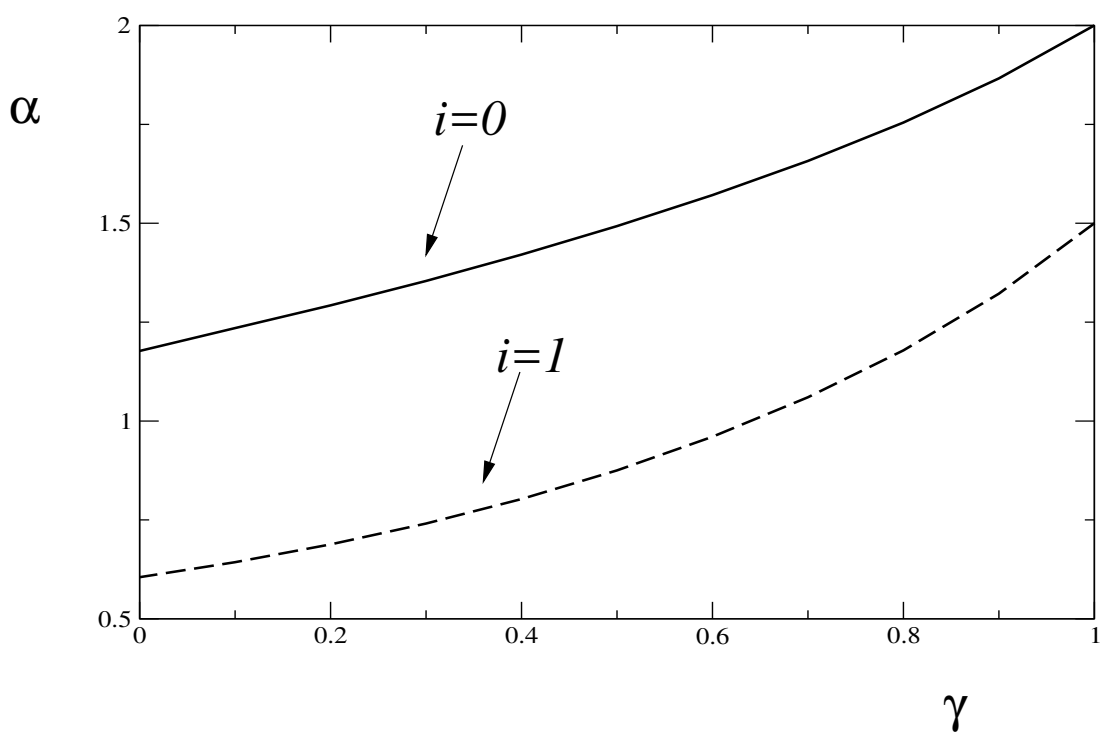

Figure 2. The exponent $\alpha$ as a function of $\gamma$. The ground state $(i=0)$ is shown as the solid line, the first unstable branch $(i=1)$ is the dashed line. For $\gamma=1, \alpha=2$ in the ground state, and $\alpha=3 / 2$ in the first unstable state.

also has to be satisfied. This is illustrated in Fig. 1 for the case $\gamma=0$ : by adjusting $\alpha$ to a critical value, the solution can be made regular at the origin, giving the approximate value $\alpha=1.177$. Clearly, this is an example of self-similarity of the second kind [17].

Based on the selection mechanism (21), we can now search for solutions of (12) directly, by finding the value of the exponent as part of the solution. The first possibility to implement this is to formulate (21) as a separate equation. However, we found this to work only if the initial condition was already very close to the correct solution. A more robust procedure is to write down a finite-difference formula for the third derivative at $\xi=0$, and to demand that $\Theta_{\xi \xi \xi}(0)=0$, using this as a separate equation.

Based on this latter idea, we employed two different approximations for $D^{(\gamma)}(\Theta)$ to obtain $\alpha$ as a function of $\gamma$, as shown in Fig. 2. For $0 \leq \gamma \leq 1 / 2$, we used (8), using the solution for $\gamma=0$ as an initial condition, and extending in small intervals of $\gamma$. For $0.1 \leq \gamma \leq 1$, we used the exact solution (20) for $\gamma=1$ as a starting point. Taking $i=0$, corresponding to the ground state, we proceed to smaller values of $\gamma$, yielding the solid line in Fig. 2. In the overlap region between the different methods there is agreement to several decimal places, so the difference is well below the line thickness in Fig. 2. If we start from the higher order solution (20) with $i=1$, a different branch is found (dashed line), which we followed down to $\gamma=0$. As seen from (19), there is an infinite sequence of exponents for $\gamma=1$, so we expect there to be an infinite sequence of unstable branches, although we explored this for the first unstable mode only. 


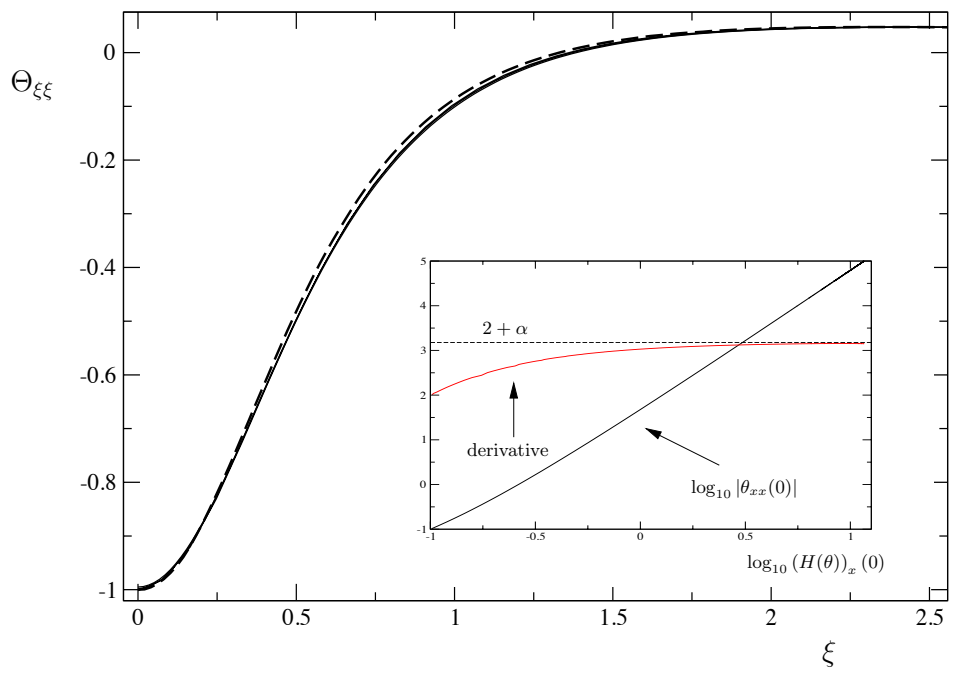

Figure 3. The similarity profile $\Theta^{\prime \prime}(\xi)$ for $\gamma=0$ (Hilbert transform). The dashed line is a solution of (12) for $\gamma=0$ and $\alpha=1.17712$. The solid lines comes from a numerical simulation of (1), rescaled according to (3), and normalised according to (13), such that $\Theta^{\prime \prime}(0)=-1$. The inset shows the convergence of the scaling exponent toward the theoretical value.

\subsection{Numerical evidence}

We performed numerical simulations of (1) using a pseudospectral method, focusing on the case $\gamma=0$ (Hilbert transform). The variable $\theta$ was assumed periodic over the interval $[0,2 \pi]$ with initial condition $\theta(x, 0)=0.1 \cos x$. The spatial grid is $x_{i}=2 \pi i / N$ for $i=0, \ldots N-1$, and the derivative $D^{(\gamma)}(\theta)$ is computed from the Fourier components

$$
\widehat{D^{(\gamma)}(\theta)}(q)=-i \operatorname{sign}(q)|q|^{\gamma} \hat{\theta}(q)
$$

for $q=1 \ldots N / 2-1$ and $\widehat{D^{(\gamma)}(\theta)}(0)=0$. At least for $\gamma=0$, this is a spectrally accurate representation of the Hilbert transform [9]. The result is transformed back into real space to obtain $D^{(\gamma)}(\theta)\left(x_{i}\right) ; \theta_{x}$ is obtained from finite differences and is treated implicitly. Simulations were performed with $N=2^{20}$ until the maximum of $\left|\theta_{x x}\right|$ reaches $10^{5}$.

As illustrated in the inset of Fig. 3, it is necessary to integrate significantly beyond a maximum curvature of $10^{3}$ in order to capture the correct asymptotic behaviour, and thus obtain a good estimate for the scaling exponent $\alpha$. We plot the logarithm of the maximum curvature $\kappa_{m}=\left|\theta_{x x}(0)\right|$, which occurs at the point of symmetry. To avoid having to first find the singularity time $t_{0}$, we plot $\log _{10} \kappa_{m}$ as a function of $\log _{10}(H(\theta))_{x}$, which scales like $t^{\prime-1}$. Since $\kappa_{m} \propto t^{\prime-2-\alpha}$, the slope should be $2+\alpha=3.177$, indicated by the horizontal dashed line. A value close to that is reached eventually, but the approach is quite slow, as seen from taking the derivative. Thus in a region of curvatures around 
$\kappa_{m} \approx 10^{3}, \alpha=1$ seems a much better approximation, which lead [12] to conjecture this value.

In the main part of Fig. 3, we compare the second derivative $\Theta_{\xi \xi}$ of the similarity profile, as obtained from solving the similarity equation, with numerical simulations of (1). First, the profile is rescaled according to (3), and then the transformation (13) is used to normalise $\Theta_{\xi \xi}(0)=-1$. We have plotted profiles (solid lines) obtained for $\kappa_{m}=10^{4}, 10^{4.5}$ and $10^{5}$. The agreement with the similarity solution (dashed line), is quite good, and the curves keep edging toward it.

\section{Moore-type singularity}

We now turn to (2), which has been studied numerically and theoretically in [12]. However, the crucial question of the selection of the scaling exponent has been left unresolved. It has been shown that the singularity is weak in that it only appears in the curvature, just like Moore's singularity of vortex sheets [29, 12, 17]. As a result, the singular part is a small contribution to a slowly varying profile, and locally we can write $\theta(x, t)=\theta_{0}+T(x, t)$, and linearize in $T$, to obtain the linear equation

$$
T_{t}=\delta \theta_{0}(H(T))_{x}
$$

Rescaling according to $x \rightarrow x \delta \theta_{0}$, we can get rid of the constant $\delta \theta_{0}$, which we disregard from now on. Taking the Hilbert transform and using that $H(H(f))=-f$, we obtain the pair of equations

$$
T_{t}=(H(T))_{x}, \quad(H(T))_{t}=-T_{x}
$$

from which we find

$$
T_{t t}+T_{x x}=0
$$

Thus small perturbations to a smooth profile are described by an elliptic equation, which shows that (2) is an ill-posed equation, and the growth rate of perturbations diverges in the limit of small wavelength. As a result, the equation can only be integrated uniquely with analytic initial data, and any numerical treatment requires some amount of smoothing $[7,30]$. Putting $z=t^{\prime}+i x$, where $t^{\prime}=t_{0}-t$ is the time distance to the singularity to be studied, solutions to (24) can be found as $T=\Re\{f(z)\}$, where $f(z)$ is an analytic function.

This allows us to find singular similarity solutions to (22), which are of the form (3) $[12,17]$, with $\beta=1$ :

$$
T(x, t)=t^{\prime \alpha} \Theta(\xi), \quad \xi=\frac{x}{t^{\prime}} ;
$$

$\alpha$ is as-yet undetermined. Inserting (25) into (22), we obtain the similarity equation

$$
-\alpha \Theta+\xi \Theta_{\xi}-(H(\Theta))_{\xi}=0
$$

As long as $\alpha>0$ (which signifies a weak singularity) $\theta=\theta_{0}+t^{\prime \alpha} \Theta(\xi)$ is also a similarity solution to (2), as terms quadratic in $\theta$ are of order $t^{\prime \alpha}$ smaller than those retained. 
Instead of solving (26) directly, we notice that $f(z)=z^{\alpha}$ leads to a similarity solution of the form (25), where

$$
\Theta(\xi)=\Re\left\{(1+i \xi)^{\alpha}\right\}=\left(1+\xi^{2}\right)^{\alpha / 2} \cos (\alpha \arctan \xi) .
$$

Note that (27) satisfies the expected growth condition $\Theta(\xi) \sim A|\xi|^{\alpha}$ as $|\xi| \rightarrow \infty$ [17], which ensures that the singularity matches to a time-independent outer solution.

By construction, $\Theta$ and $H(\Theta)$ form a Cauchy-Riemann pair, and thus

$$
H(\Theta)=\Im\left\{(1+i \xi)^{\alpha}\right\}=\left(1+\xi^{2}\right)^{\alpha / 2} \sin (\alpha \arctan \xi) .
$$

Using (28), it is easy to check that (27) indeed solves the similarity equation (26). Interestingly, Moore's singularity of vortex sheets is identical to (27) (cf. [13]), if $\Theta$ is taken as the slope of the vortex sheet. Another problem in which the same singularity appears is in the small dispersion limit of the focusing nonlinear Schrödinger equation [14]. In the vortex sheet problem, the selection of $\alpha$ remains unresolved, but numerical evidence points to $\alpha=1 / 2$, in which case (27) assumes the form

$$
\Theta(\xi)=\Re\{\sqrt{1+i \xi}\}=\frac{1}{\sqrt{2}}\left(1+\sqrt{1+\xi^{2}}\right)^{1 / 2} .
$$

In the Schrödinger problem, the same exponent 1/2 (which corresponds to an ellipticumbilic singularity of catastrophe theory [1]) is selected once more as a result of taking the singular limit.

To address the selection problem, we note that (28) is a solution of the linearized problem, and is thus insensitive to the specific form of (2). In particular, in [12] it was shown numerically that $\alpha$ depends on the parameter $\delta$. We will show that the selection of $\alpha$ depends on the way the similarity solution (27) is approached. Thus we consider the first correction to $(27)$, which is of order $t^{\prime 2 \alpha}$ :

$$
\theta(\xi, t)=\theta_{0}+t^{\prime \alpha} \Theta(\xi)+t^{\prime 2 \alpha} G(\xi, \tau)
$$

where $\Theta(\xi)$ is given by (27) and $\tau=-\log t^{\prime}$. Inserting (30) into (2), we obtain

$$
G_{\tau}-2 \alpha G+\xi G_{\xi}-H\left(G_{\xi}\right)=F(\xi)+N L[G]
$$

where

$$
F(\xi) \equiv \frac{1}{\delta \theta_{0}}\left[\delta(\Theta H(\Theta))_{\xi}+(1-\delta) H(\Theta) \Theta_{\xi}\right]
$$

and $N L[G]$ is a nonlinear (in fact, the sum of a linear and a quadratic) operator of $G$.

Let us now consider solving (31) as a fixed point for a mapping $\mathbb{T}$, which assigns $\bar{G}$ in a certain class of functions to the solution $G$ of (31), with $\bar{G}$ replacing $G$ at the right hand side. A necessary condition for such a fixed point to exist is that $\mathbb{T}$ maps the class into itself. Hence, if we start with $\bar{G}$ having a certain growth as $\xi$ tends to infinity, it is necessary that the resulting $G$ presents the same or lower growth. 
If we neglect, as a first order approximation, the nonlinearity at the right hand side of (31), we seek $\tau$-independent solutions solving

$$
-2 \alpha G+\xi G_{\xi}-H\left(G_{\xi}\right)=F(\xi)
$$

The generic behaviour of $G(\xi)$ at large $\xi$ is $A_{2} \xi^{2 \alpha}$, which means that $G$ grows faster than $\Theta$. Instead, for the last term at the right hand side of (30) to be a uniformly small perturbation, we require that $A_{2}=0$.

To calculate $F$, we note that

$$
2(\Theta H(\Theta))_{\xi}=\Im\left\{(1+i \xi)^{2 \alpha}\right\}_{\xi}=2 \alpha \Re\left\{(1+i \xi)^{2 \alpha-1}\right\}, \quad \Theta_{\xi}=-\alpha \Im\left\{(1+i \xi)^{\alpha-1}\right\}
$$

and so

$$
\begin{aligned}
& F(\xi)=\alpha\left(1+\xi^{2}\right)^{\alpha-1 / 2}[\delta \cos ((2 \alpha-1) \arctan \xi)+ \\
& (1-\delta) \sin ((\alpha-1) \arctan \xi)) \cos (\alpha \arctan \xi)] \\
= & \alpha\left(1+\xi^{2}\right)^{\alpha-\frac{1}{2}}\left[\frac{1+\delta}{2} \cos ((2 \alpha-1) \arctan \xi)-\frac{1-\delta}{2}\left(1+\xi^{2}\right)^{-1 / 2}\right] .
\end{aligned}
$$

Taking the Fourier transform of (32), we find

$$
-2 \alpha \widehat{G}-k \frac{d \widehat{G}}{d k}+|k| \widehat{G}=\widehat{F},
$$

which for $k>0$ leads to the solution

$$
\widehat{G}(k)=e^{|k|} k^{-(2 \alpha+1)} \int_{k}^{\infty} e^{-\left|k^{\prime}\right|} k^{\prime 2 \alpha} \widehat{F}\left(k^{\prime}\right) d k^{\prime} .
$$

The factor $k^{-(2 \alpha+1)}$, which is singular at the origin, implies a power-law growth as $\xi^{2 \alpha}$ for $G(\xi)$ as $\xi \rightarrow \infty$. In order to avoid such a growth we impose

$$
\operatorname{Re} \int_{0}^{\infty} e^{-\left|k^{\prime}\right|} k^{\prime 2 \alpha} \widehat{F}\left(k^{\prime}\right) d k^{\prime}=0
$$

which, performing the Fourier transform of $F(\xi)$, is

$$
\operatorname{Re} \int_{0}^{\infty} e^{-\left|k^{\prime}\right|} k^{\prime 2 \alpha}\left[\int_{-\infty}^{\infty} e^{i k^{\prime} \xi}\left(\frac{1+\delta}{2} \operatorname{Re}(1+\xi i)^{2 \alpha-1}-\frac{1-\delta}{2}\left(1+\xi^{2}\right)^{\alpha-1}\right) d \xi\right] d k^{\prime}=0
$$

Exchanging the integrals and using

$$
\int_{0}^{\infty} e^{-\left|k^{\prime}\right|} k^{\prime 2 \alpha} e^{i k^{\prime} \xi} d k^{\prime}=\frac{\Gamma(2 \alpha+1)}{(1-i \xi)^{2 \alpha+1}}
$$

we obtain

$$
\operatorname{Re} \int_{-\infty}^{\infty} \frac{1}{(1-i \xi)^{2 \alpha+1}}\left(\frac{1+\delta}{2} \operatorname{Re}(1+\xi i)^{2 \alpha-1}-\frac{1-\delta}{2}\left(1+\xi^{2}\right)^{\alpha-1}\right) d \xi=0,
$$




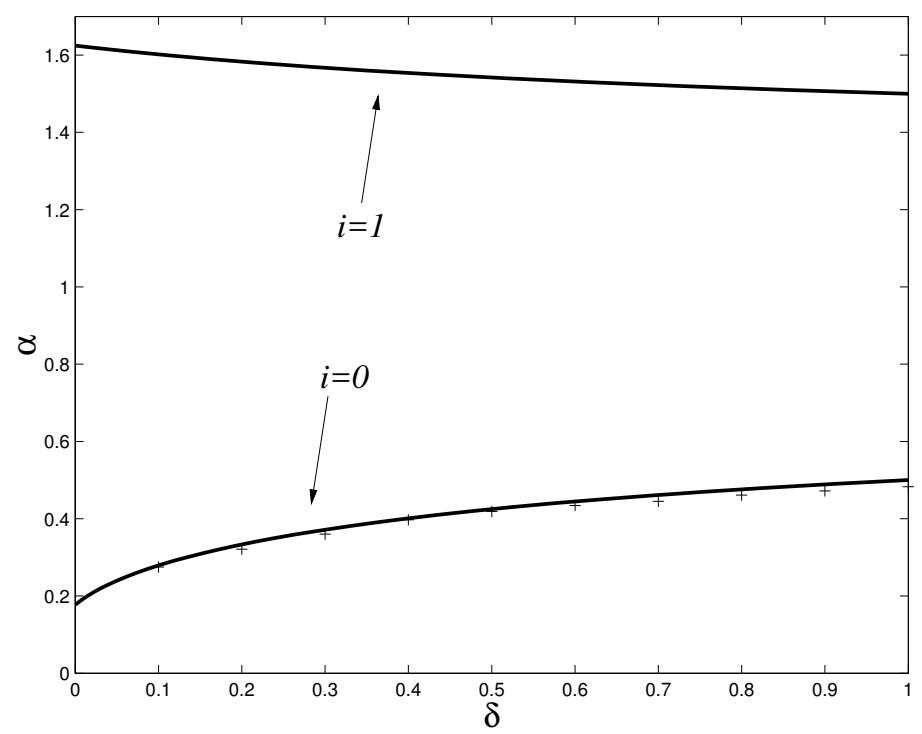

Figure 4. The first two branches of exponents $\alpha$ as described by (36). The lower branch, corresponding to the ground state $i=0$, is compared to values obtained numerically from a solution of (2) (crosses).

and hence

$$
\begin{aligned}
& \int_{-\infty}^{\infty}\left(\frac{1+\delta}{2} \frac{1}{1+\xi^{2}} \cos ((2 \alpha+1) \arctan \xi) \cos (2 \alpha-1) \arctan \xi\right)- \\
& \left.\frac{1-\delta}{2} \frac{1}{\left(1+\xi^{2}\right)^{\frac{3}{2}}} \cos ((2 \alpha+1) \arctan \xi)\right) d \xi=0,
\end{aligned}
$$

after taking the real part. The substitution $u=\arctan \xi$ yields

$$
\left.\int_{-\frac{\pi}{2}}^{\frac{\pi}{2}}\left(\frac{1+\delta}{2} \cos ((2 \alpha+1) u) \cos (2 \alpha-1) u\right)-\frac{1-\delta}{2} \cos u \cos ((2 \alpha+1) u)\right) d u=0
$$

which leads to

$$
\frac{1+\delta}{2} \frac{\sin (2 \alpha \pi)}{4 \alpha}-\frac{1-\delta}{2}\left(\frac{\sin ((\alpha+1) \pi)}{2 \alpha+2}+\frac{\sin (\alpha \pi)}{\alpha}\right)=0
$$

or, simplifying,

$$
(\alpha+1) \cos (\alpha \pi)=\frac{1-\delta}{1+\delta} .
$$

Equation (36) is the desired relation which selects the similarity exponent $\alpha$ as a function of the parameter $\delta$, as shown in Fig. 4. For $\delta=1$, one obtains the sequence of solutions $\alpha=(2 i+1) / 2$, with $i$ a non-negative integer. The ground state solution $i=0$ corresponds to a generic elliptic-umbilic singularity [14], while the higher order solutions with $i>0$ are expected to be unstable, in analogy with the Burgers-type equation (1) we analysed before. 
As seen in Fig. 4, each of these solution branches continues to $\delta=0$, but the value of $\alpha$ is no longer rational. The lowest $(i=0)$ branch is compared to exponents obtained numerically from integrating (2), as explained in detail in Appendix B. The numerical $\alpha$-values are shown as pluses, and are seen to agree well with the lowest branch of (36). This confirms that this branch indeed corresponds to a stable similarity solution.

\section{Discussion}

In this paper, we revealed the mechanism of exponent selection for two non-local, nonlinear transport equations. As a function of parameters $\gamma$ and $\delta$, respectively, both equations extend exactly solvable cases for $\gamma=1$ and $\delta=1$ to new branches of solutions. In the case of (1), for $\gamma=1$ the equation can be reduced to Burgers' equation, in the case of (2) it is complex Burgers' equation for $\delta=1$. Using exact solutions to these equations, scaling exponents can be deduced based on genericity arguments $[17,20]$. It is found that apart from the generic, stable solution, there is an infinite sequence of non-generic, unstable solutions, which can only be reached for a specific choice of initial conditions. Since the local scaling is obtained from local expansions into power series, the exponents assume rational values.

However, as the solution branches are continued to arbitrary $\gamma$ and $\delta$, we have seen that the scaling exponents assume arbitrary irrational values. Hence the arguments advanced previously for the cases $\gamma=\delta=1$ no longer apply, and new mechanisms for exponent selection have to be found. In the case of the Burgers-type equation (1), selection is described by condition (21), which ensures that the similarity solution is regular at the origin.

In the case of the Moore-type equation (2), the shape of the similarity solution is determined by the linearized equation (22). However, the linearized equation does not contain a mechanism for the selection of the exponent. Instead, we have shown that the complex analytic structure of the equation (complex Burger's equation for $\delta=1$, as

shown in [8]), including leading nonlinear terms, is essential for selection in Moore-type singularities.

This fact was already recognised in [5] and [6] for the case of the complex Burgers equation, the Birkhoff-Rott equation for vortex sheets, as well as more general systems. Other equations and systems, leading to the same linear equation but with more general nonlinearities, generate singularities with different exponents, a fact that was shown numerically in [12] for the present equation and in [21] for the case of vortex sheets separating fluids with different and nonzero densities. In our analysis of (2), we were finally able to handle the non-linear part of the equation analytically, and to compute the exponent using the non-linear structure of the equation. We hope this will be a starting point to apply a similar analysis to Moore's problem itself. 


\section{Appendix A. Asymptotics for large arguments}

Here we consider the behaviour of (12) for large arguments of $\Theta$. To this end, we consider the asymptotics of $D^{(\gamma)}(\Theta)(\xi)$ for large $\xi$, and take a function $f$ such that for $y>A$ we can approximate $f(y) \approx y^{\nu}$. Then using (10), we can split the integral into two parts (assuming $x \gg A$ ):

$$
\begin{gathered}
D^{(\gamma)}(f)(x)=\frac{P_{\gamma} x^{-\gamma}}{\gamma} \int_{0}^{A} f^{\prime}(y)\left[\left(1+\frac{y}{x}\right)^{-\gamma}-\left(1-\frac{y}{x}\right)^{-\gamma}\right] d y+ \\
\frac{P_{\gamma} \nu x^{-\gamma}}{\gamma} \int_{A}^{\infty} y^{\nu-1}\left[\left(1+\frac{y}{x}\right)^{-\gamma}-\left|1-\frac{y}{x}\right|^{-\gamma}\right] d y .
\end{gathered}
$$

In the first integral, we can approximate the term in square brackets as []$\approx-2 \gamma y / x$, in the second integral, we substitute $t=y / x$, so we find

$$
D^{(\gamma)}(f)(x)=-2 P_{\gamma} x^{-\gamma-1} \int_{0}^{A} f^{\prime}(y) y d y+\frac{P_{\gamma} \nu x^{\nu-\gamma}}{\gamma} \int_{0}^{\infty} t^{\nu-1}\left[(1+t)^{-\gamma}-|1-t|^{-\gamma}\right] d t .
$$

But this means that

$$
D^{(\gamma)}(f)(x)=B x^{\nu-\gamma}+O\left(x^{-\gamma-1}\right), \quad B(\nu, \gamma)=\frac{P_{\gamma} \nu}{\gamma} \int_{0}^{\infty} t^{\nu-1}\left[(1+t)^{-\gamma}-|1-t|^{-\gamma}\right] d t
$$

The constant $B$ can be calculated (putting $\delta=\gamma-\nu$ ) in terms of hypergeometric functions:

$$
\begin{aligned}
& B(\nu, \gamma)=\left[-\Gamma(\nu-\gamma) \sin (\pi \delta)^{2} \nu F(\gamma, \delta ; \gamma-\nu+1,-1)+\right. \\
& (-F(\gamma, \nu ; 1+\nu,-1) \sin (\pi \delta) \delta \Gamma(\nu-\gamma) \gamma \\
& \Gamma(-\gamma) \Gamma(1+\nu)(-\sin (\pi \delta)+\sin (\pi \gamma))) \sin (\pi \delta)- \\
& \Gamma(1+\nu) \gamma \Gamma(-\gamma)(\cos (\pi \nu)+\cos (\pi \gamma))(\cos (\pi \delta)-1)] \\
& {\left[-2 \Gamma(-\gamma) \gamma \Gamma(\nu-\gamma) \sin (\pi \delta)^{2} \delta \sin (\pi \gamma / 2)\right]}
\end{aligned}
$$

For $\gamma=1, D^{(\gamma)}(f)(x)=f^{\prime}(x)$, and so $B(\nu, 1)=-\nu$.

The leading order behaviour $\Theta \propto \xi^{\nu}$ cancels the right hand side of (12), which corresponds to the usual matching condition, which requires the time derivative to cancel far from the singularity. Then the right hand side scales like $\xi^{2 \nu-\gamma-1}$; balancing this with the left hand side of (12), the expansion (15) results.

\section{Appendix B. Numerical solution of (2)}

We use a spectral method, writing the profile as a cosine series:

$$
\theta(x, t)=\sum_{n=0}^{\infty} a_{n} \cos (n x), \quad a_{n}=a_{n}(t)
$$


It is then straightforward to compute

$$
\begin{aligned}
((H(\theta)) \theta)_{x} & =\left(\left(\sum_{n=0}^{\infty} a_{n} \sin (n x)\right)\left(\sum_{m=0}^{\infty} a_{m} \cos (m x)\right)\right)_{x} \\
& =\frac{1}{2} \sum_{n, m} a_{n} a_{m}((n+m) \cos (n+m-(n-m) \cos (n-m) x) x) \\
& =\frac{1}{2} \sum_{m=0}^{\infty}\left(\sum_{j=m}^{\infty} a_{m} a_{j-m} j \cos j x-\sum_{j=-m}^{\infty} a_{m} a_{m+j} j \cos j x\right) \\
& =\sum_{j=0}^{\infty}\left(\sum_{m=0}^{\infty} \frac{j}{2}\left(a_{m} a_{|m-j|}-a_{m} a_{m+j}\right)\right) \cos j x
\end{aligned}
$$

as well as

$$
\begin{aligned}
& (H(\theta)) \theta_{x}=-\left(\sum_{n=0}^{\infty} a_{n} \sin (n x)\right)\left(\sum_{m=0}^{\infty} a_{m} m \sin (m x)\right) \\
= & -\sum_{n, m} a_{n} a_{m} \frac{m}{2}(\cos (m-n) x-\cos (m+n) x) \\
= & -\sum_{m=0}^{\infty} \sum_{j=-m}^{\infty} a_{m+j} a_{m} \frac{m}{2} \cos (j x)+\sum_{m=0}^{\infty} \sum_{j=m}^{\infty} a_{j-m} a_{m} \frac{m}{2} \cos (j x) \\
= & -\sum_{m=0}^{\infty} \sum_{j=0}^{m} a_{m-j} a_{m} \frac{m}{2} \cos (j x)-\sum_{m=0}^{\infty} \sum_{j=1}^{\infty} a_{m+j} a_{m} \frac{m}{2} \cos (j x)+\sum_{m=0}^{\infty} \sum_{j=m}^{\infty} a_{j-m} a_{m} \frac{m}{2} \cos (j x) \\
= & -\sum_{m=0}^{\infty} \frac{m}{2} a_{m}^{2}+\sum_{j=1}^{\infty}\left(\sum_{m=0}^{\infty}\left(\operatorname{sign}(j-m) a_{|m-j|} a_{m}-a_{m+j} a_{m}\right) \frac{m}{2}\right) \cos (j x) .
\end{aligned}
$$

Comparing coefficients, we can write (2) as an infinite system of ODE's (one for each mode $\cos n x)$ :

$$
a_{0, t}=-(1-\delta) \sum_{m=0}^{\infty} \frac{m}{2} a_{m}^{2}
$$

and, for $j \geq 1$

$$
\begin{aligned}
a_{j, t}= & \delta\left(\sum_{m=0}^{\infty} \frac{j}{2}\left(a_{m} a_{|m-j|}-a_{m} a_{m+j}\right)\right) \\
& +(1-\delta)\left(\sum_{m=0}^{\infty}\left(\operatorname{sign}(j-m) a_{|m-j|} a_{m}-a_{m+j} a_{m}\right) \frac{m}{2}\right) .
\end{aligned}
$$

We solved the ODE system (B.2),(B.3), which was truncated at $j=10^{4}$ modes, using an explicit time integrator. As initial data we took $\theta(x, 0)=\cos (x)$. The maximum of $\theta$ occurs at $x=2 n \pi$, which is where the curvature achieves its maximum as well:

$$
\kappa_{m} \equiv\left|\theta_{x x}\right|(0)=\sum_{j=1}^{\infty} j^{2} a_{j}
$$


Taking the second derivative of (25), one finds that $\kappa_{m} \propto t^{\prime \alpha-2}$, which means that $\kappa_{m}^{1 /(2-\alpha)}$ is a linear function $t^{\prime}=t_{0}-t$.

We found $\alpha$ numerically by adjusting the value of $\alpha$ such that $\kappa_{m}^{1 /(2-\alpha)}$ as a function of time is approximated by a straight line with minimum error. For better accuracy, we considered at least two decades of $\kappa_{m}$, typically $\kappa_{m} \in\left(10^{2}, 10^{4}\right)$. For the optimal values

of $\alpha$, we found the residual in a linear least-square fit of $\kappa_{m}^{1 /(2-\alpha)}$ vs. time to be less than $10^{-3}$ in all cases.

\section{References}

[1] V. I. Arnold, Catastrophe theory, Springer, 1984.

[2] C. Bardos and D. Lannes, Mathematics for 2d interfaces, Panoramas et Synthèses 38 (2013), $37-67$.

[3] G. I. Barenblatt, Similarity self-similarity and intermedeate asymptotics, Cambridge University Press, Cambridge, 1996.

[4] G. I. Barenblatt and Y. B. Zel'dovich, Self-similar solutions as intermediate asymptotics, Annu. Rev. Fluid Mech. 4 (1972), 285-312.

[5] R. Caflisch and S. Semmes, A nonlinear approximation for vortex sheet evolution and singularity formation, Physica D 41 (1990), 197-207.

[6] R. E. Caflisch, N. Ercolani, T. Y. Hou, and Y. Landis, Multi-valued solutions and branch point singularities for nonlinear hyperbolic and elliptic systems, Comm. Pure and Appl. Math. 46 (1993), 453-499.

[7] R. E. Caflisch and O. F. Orellana, Singular solutions and ill-posedness for the evolution of vortex sheets, SIAM J. Math. Anal. 20 (1989), 293-307.

[8] D. Chae, A. Córdoba, D. Córdoba, and M. A. Fontelos, Finite time singularities in a $1 d$ model of the quasi-geostrophic equation, Advances in Mathematics 194 (2005), 203-223.

[9] V. Čížek, Discrete Hilbert transform, IEEE Trans. Audio Electroacoust. 18 (1970), 340.

[10] P. Constantin, P. D. Lax, and A. Majda, A simple onedimensional model for the threedimensional vorticity equation, Comm. Pure Appl. Math. 38 (1985), 715-724.

[11] A. Córdoba, D. Córdoba, and M. A. Fontelos, Formation of singularities for a transport equation with nonlocal velocity, Ann. of Math. 162 (2005), 1375-1387.

[12] F. de la Hoz and M. A. Fontelos, The structure of singularities in nonlocal transport equations, J. Phys. A 41 (2008), 185204.

[13] F. de la Hoz, M. A. Fontelos, and L. Vega, The effect of surface tension on the Moore singularity of vortex sheet dynamics, J. Nonlin. Sci. 18 (2008), 463-484.

[14] B. Dubrovin, T. Grava, and C. Klein, On universality of critical behaviour in the focusing nonlinear Schrödinger equation, elliptic umbilic catastrophe and the tritronquée solution to the Painlevé equation, J. Nonlin. Sci. 19 (2009), 57-94.

[15] J. Duchon and R. Robert, Global vortex sheet solution of euler equations in the plane, J. Differential Equations 73 (1988), 215-224.

[16] J. Eggers and M. A. Fontelos, The role of self-similarity in singularities of partial differential equations, Nonlinearity 22 (2009), R1-R44.

[17] - Singularities: Formation, structure, and propagation, Cambridge University Press, Cambridge, 2015.

[18] J. Eggers, T. Grava, M. A. Herrada, and G. Pitton, Spatial structure of shock formation, J. Fluid Mech. 820 (2017), 208-231.

[19] J. Eggers and J. Hoppe, Singularity formation for timelike extremal hypersurfaces, Phys. Lett. B $680(2009), 274$. 
[20] J. Eggers and N. Suramlishvili, Singularity theory of plane curves and its applications, Eur. J. Mech. B 65 (2017), 107-131.

[21] M. A. Fontelos and F. de la Hoz, Singularities in water waves and the Rayleigh-Taylor problem, J. Fluid Mech. 651 (2010), 211-239.

[22] A. Kiselev, Regularity and blow up for active scalars, Math. Model. Nat. Phenom. 5 (2010), 225255.

[23] R. Krasny, A study of singularity formation in a vortex sheet by the point-vortex approximation, J. Fluid Mech. 167 (1986), 65-93.

[24] E. A. Kuznetsov, M.D. Spector, and V. E. Zakharov, Surface singularities of ideal fluid, Phys. Lett. A 182 (1993), 387.

[25] E. A. Kuznetsov and N. M. Zubarev, Singularity formation on a fluid interface during the kelvinhelmholtz instability development, JETP 119 (2014), 169.

[26] L. D. Landau and E. M. Lifshitz, Fluid mechanics, Pergamon: Oxford, 1984.

[27] A. J. Majda and A. L. Bertozzi, Vorticity and incompressible flow, Cambridge University Press, Cambridge, 2002.

[28] I. Meiron, G. R. D. Baker, and S. A. Orszag, Analytic structure of vortex sheet dynamics. part 1. Kelvin-Helmholtz instability, J. Fluid Mech. 114 (1982), 283-298.

[29] D. W. Moore, Spontaneous appearance of a singularity in the shape of an evolving vortex sheet, Proc. Roy. Soc. London A 365 (1979), 105-119.

[30] M. A. Page and S. J. Cowley, On the formation of small-time curvature singularities in vortex sheets, IMA J. Appl. Math. 83 (2018), 188-203.

[31] Y. Pomeau, M. Le Berre, P. Guyenne, and S. Grilli, Wave-breaking and generic singularities of nonlinear hyperbolic equations, Nonlinearity 21 (2008), T61-T79. 